NTERnational Electronic Journal of Algebra

Volume 29 (2021) 148-164

DOI: $10.24330 /$ ieja.852139

\title{
J-IDEALS OF COMMUTATIVE RINGS
}

\author{
Hani A. Khashan and Amal B. Bani-Ata \\ Received: 27 February 2020; Revised: 7 June 2020; Accepted: 15 June 2020 \\ Communicated by Abdullah Harmancı
}

\begin{abstract}
Let $R$ be a commutative ring with identity and $N(R)$ and $J(R)$ denote the nilradical and the Jacobson radical of $R$, respectively. A proper ideal $I$ of $R$ is called an n-ideal if for every $a, b \in R$, whenever $a b \in I$ and $a \notin N(R)$, then $b \in I$. In this paper, we introduce and study J-ideals as a new generalization of n-ideals in commutative rings. A proper ideal $I$ of $R$ is called a J-ideal if whenever $a b \in I$ with $a \notin J(R)$, then $b \in I$ for every $a, b \in R$. We study many properties and examples of such class of ideals. Moreover, we investigate its relation with some other classes of ideals such as r-ideals, prime, primary and maximal ideals. Finally, we, more generally, define and study J-submodules of an $R$-modules $M$. We clarify some of their properties especially in the case of multiplication modules.
\end{abstract}

Mathematics Subject Classification (2020): 13A15, 13A18, 13A99

Keywords: n-ideal, r-ideal, J-ideal, J-submodule

\section{Introduction}

We assume throughout this paper that all rings are commutative with identity. By a proper ideal $I$ of a ring $R$ we mean an ideal $I \neq R$. For a ring $R$, the set of units in $R$, the nil radical of $R$, the Jacobson radical of $R$ and the set of zero divisors in $R$ are denoted by $U(R), N(R), J(R)$ and $Z(R)$ respectively. For undefined notations or terminologies in commutative ring theory, we refer the reader to [11].

Over the years, several types of ideals have been developed in order to let us fully understand the structures of rings in general. Examples of such types include prime, primary and maximal ideals which play a key role in the theory of commutative algebra. In the last three decades, many generalizations and related types of such ideals have been studied such as weakly prime (primary), almost prime (primary), $\varphi$-prime (primary) and semi-prime, see [1], [5], [6], [7], [9].

Recently, [10] Rostam Mohamadian has introduced the concept of r-ideals in commutative rings. A proper ideal $I$ of a ring $R$ is called an r-ideal if whenever $a, b \in R$ with $a b \in I$ and $A n n(a)=0$, then $b \in I$ where $A n n(a)=\{r \in R: r a=0\}$. He investigated the behavior of r-ideals and compare them with other classical ideals 
such as prime and maximal ideals. In [13], Tekir et al., defined and studied some subclass of r-ideals, namely, the class of n-ideals. A proper ideal $I$ of a ring $R$ is called an n-ideal if whenever $a, b \in R$ with $a b \in I$ and $a \notin N(R)$, then $b \in I$.

In the second section of this paper, we continue to pursue this approach and present J-ideals as a generalization of n-ideals. A proper ideal $I$ of a ring $R$ is called a J-ideal if whenever $a, b \in R$ with $a b \in I$ and $a \notin J(R)$, then $b \in I$. We will first justify that this generalization is proper by giving examples of J-ideals which are not n-ideals in certain rings. Indeed, we prove that while every proper ideal in a quasilocal ring $R$ is a J-ideal, the ideal $J(R)$ is not an n-ideal if $N(R) \neq J(R)$. We also give some examples to show that in general J-ideals are not comparable with prime, maximal and r-ideals. However, if a ring $R$ is presimplifiable $(Z(R) \subseteq J(R))$, then we prove that any r-ideal in $R$ is a J-ideal. In particular, if a ring $R$ is balanced domainlike $(R \backslash Z(R) \subseteq U(R)$ and $Z(R) \subseteq N(R))$, then the classes of n-ideals, r-ideals and J-ideals are coincide. Next, we give several characterizations of J-ideals and Proposition 2.10 is devoted to some of such characterizations. We, moreover, clarify that an ideal of a ring contained in the Jacobson radical is a J-ideal if and only if it is J-primary.

Among many other results in this section, it is shown (Proposition 2.18) that whenever $R$ is a ring and $I$ is a proper ideal of $R$, then $I[|x|]$ is a J-ideal of $R[|x|]$ if and only if $I$ is a J-ideal of $R$. For a multiplicatively closed subset $S$ of a ring $R$, we discuss in Proposition 2.26 certain conditions under which the extension (contraction) of a J-ideal of $R$ (of $S^{-1} R$ ) is a J-ideal of $S^{-1} R$ (of $R$ ).

For a ring $R$, an $R$-module $M$ and a submodule $N$ of $M$, we set $(N: M)=$ $\{r \in R: r m \in N$ for all $m \in M\}$. This set is an ideal of $R$ called the residual ideal of $N$ in $M$. For an ideal $I$ of $R$, it is well-known that the set $I M=$ $\left\{\sum_{i=1}^{n} a_{i} m_{i}: a_{i} \in I, m_{i} \in M, n \in \mathbb{N}\right\}$ is a submodule of $M$. More generally, in Section 3 , we define and study J-submodules of $R$-modules. A proper submodule $N$ of an $R$-module $M$ is called a $\mathrm{J}$-submodule of $M$ if for $a \in R$ and $m \in M$, whenever $a m \in N$ and $a \notin(J(R) M: M)$, then $m \in N$. By Nakayama's Lemma, if $M$ is a finitely generated $R$-module, then the ideal $(J(R) M: M)$ is proper. In the case $(J(R) M: M)=R$, it is clear by the definition that every submodule of $M$ is a J-submodule. We investigate many properties and examples concerning the class of J-submodules. As a special case, we study such submodules in multiplication $R$-modules $M$ where every submodule is of the form $I M$ for some ideal $I$ of $R$.

Finally, for an $R$-module $M$, we consider the idealization $\operatorname{ring} R(M)$ of $M$ in $R$. Let $I$ be an ideal of $R$ and $N$ be a submodule of $M$ such that $I M \subseteq N$. We 
prove (Proposition 3.14) that $I$ is a J-ideal and $N$ is a J-submodule whenever the homogeneous ideal $I(N)$ of $R(M)$ is a J-ideal. We also justify some conditions for which the converse is true (Proposition 3.12).

\section{J-ideals in commutative rings}

In this section, we introduce the class of J-ideals and investigate some of their properties. Moreover, we clarify their relation with some other types of ideals such as n-ideals, r-ideals, prime and maximal ideals.

Definition 2.1. A proper ideal $I$ of a ring $R$ is called a $J$-ideal if whenever $a, b \in R$ with $a b \in I$ and $a \notin J(R)$, then $b \in I$.

Proposition 2.2. If $I$ is a J-ideal of a ring $R$, then $I \subseteq J(R)$.

Proof. Suppose $I$ is a J-ideal but $I \nsubseteq J(R)$. Then there exists $a \in I$ such that $a \notin J(R)$. Now $a \cdot 1 \in I$ and $a \notin J(R)$ implies that $1 \in I$, a contradiction. Hence, $I \subseteq J(R)$ as needed.

However, we can find an ideal $I$ of a ring $R$ with $I \subseteq J(R)$ which is not a J-ideal. For example, consider the ring $\mathbb{Z}_{36}$. Then $J\left(\mathbb{Z}_{36}\right)=\langle\overline{6}\rangle$ and $I=\langle\overline{12}\rangle \subseteq J\left(\mathbb{Z}_{36}\right)$. But $I$ is not a J-ideal since $\overline{3} . \overline{4} \in I$ with $\overline{3} \notin J\left(\mathbb{Z}_{36}\right)$ and $\overline{4} \notin I$.

Next, we characterize rings in which every proper ideal is a J-ideal.

Proposition 2.3. Let $R$ be a ring. The following are equivalent.

(1) $R$ is a quasi-local ring.

(2) Every proper ideal of $R$ is a J-ideal.

(3) Every proper principal ideal of $R$ is a J-ideal.

Proof. (1) $\Rightarrow(2)$ Let $I$ be an ideal and $a, b \in R$ with $a b \in I$ and $a \notin J(R)=M$ (the unique maximal ideal of $R$ ). Then $a \in U(R)$ and so $a^{-1}(a b) \in I$. Therefore, $b \in I$ and $I$ is a J-ideal.

$(2) \Rightarrow(3)$ Clear.

$(3) \Rightarrow(1)$ Suppose that every proper principal ideal of $R$ is a J-ideal. Let $M$ be a maximal ideal of $R$ and let $a \in M$. Now, $\langle a\rangle$ is a J-ideal and 1.a $\in\langle a\rangle$. If $a \notin J(R)$, then $1 \in\langle a\rangle$, a contradiction. Thus, $a \in J(R)$ and $M=J(R)$ is the unique maximal ideal of $R$. Therefore, $R$ is a quasi-local ring.

Proposition 2.4. In any ring $R$, every $n$-ideal $I$ of $R$ is a J-ideal.

Proof. Let $a, b \in R$ such that $a b \in I$ and $a \notin J(R)$. Since $N(R) \subseteq J(R)$, $a \notin N(R)$ and so $b \in I$ as $I$ is an n-ideal. Therefore, $I$ is a J-ideal of $R$. 
However, the converse of Proposition 2.4 is not true in general as we can see in the following example.

Example 2.5. Consider the quasi-local ring $\mathbb{Z}_{\langle 2\rangle}=\left\{\frac{a}{b}: a, b \in \mathbb{Z}, 2 \nmid b\right\}$. Since any ideal in $\mathbb{Z}_{\langle 2\rangle}$ is a J-ideal by Proposition 2.3, $\langle 6\rangle_{\langle 2\rangle}=\left\{\frac{a}{b}: a \in\langle 6\rangle, 2 \nmid b\right\}$ is a J-ideal of $\mathbb{Z}_{\langle 2\rangle}$ which is not an n-ideal. Indeed, we have for example, $\frac{2}{3}, \frac{3}{5} \in \mathbb{Z}_{\langle 2\rangle}$ and $\frac{2}{3} \cdot \frac{3}{5}=\frac{6}{15} \in\langle 6\rangle_{\langle 2\rangle}$ but $\frac{2}{3} \notin N\left(\mathbb{Z}_{\langle 2\rangle}\right)=\{0\}$ and $\frac{3}{5} \notin\langle 6\rangle_{\langle 2\rangle}$.

The previous example is a special case of the following proposition.

Proposition 2.6. Let $R$ be a quasi-local ring for which $N(R) \varsubsetneqq J(R)$. Then $J(R)$ is a J-ideal which is not an n-ideal of $R$.

Proof. Since $R$ is a quasi-local ring, $J(R)$ is a J-ideal by Proposition 2.3. Choose $a \in J(R) \backslash N(R)$. Then $a=a .1 \in J(R)$ where $a \notin N(R)$ and $1 \notin J(R)$. Therefore, $J(R)$ is not an n-ideal of $R$.

In particular, the formal power series ring $F[[x]]$ over any field $F$ is a quasi-local ring for which $N(F[[x]])=\{0\} \varsubsetneqq\langle x\rangle=J(F[[x]])$. Therefore, $\langle x\rangle$ is a J-ideal that is not an n-ideal in $F[[x]]$.

We recall that a ring $R$ is called a Jacobson ring if $J(R)=N(R)$. It is clear that n-ideals and J-ideals coincide in Jacobson rings.

In general, the class of J-ideals is not comparable with the classes of r-ideals, prime ideals and maximal ideals.

Example 2.7. (1) Consider the ring $\mathbb{Z}_{10}$. Then the ideal $\langle\overline{5}\rangle$ is a prime (maximal) ideal and an r-ideal of $\mathbb{Z}_{10}$. Indeed, if for nonzero $a, b \in \mathbb{Z}_{10}, a b \in\langle\overline{5}\rangle$ and $\operatorname{Ann}(a)=0$, then $a$ is a unit and so $b \in\langle\overline{5}\rangle$. On the other hand, $\langle\overline{5}\rangle$ is not a J-ideal of $\mathbb{Z}_{10}$ as $\langle\overline{5}\rangle \nsubseteq J\left(\mathbb{Z}_{10}\right)=\langle\overline{0}\rangle$.

(2) The ring $\mathbb{Z}_{8}$ is a quasi-local ring, and so the ideal $\langle\overline{4}\rangle$ is a J-ideal of $\mathbb{Z}_{8}$ that is not prime (maximal).

(3) The ideal $\langle 6\rangle_{\langle 2\rangle}$ is a J-ideal of the quasi-local ring $\mathbb{Z}_{\langle 2\rangle}=\left\{\frac{a}{b}: a, b \in \mathbb{Z}, 2 \nmid b\right\}$ that is not an r-ideal. For example, $\frac{3}{5}, \frac{2}{3} \in \mathbb{Z}_{\langle 2\rangle}$ with $\frac{3}{5} \cdot \frac{2}{3}=\frac{6}{15} \in\langle 6\rangle_{\langle 2\rangle}$ and $\operatorname{Ann}\left(\frac{2}{3}\right)=0$ but $\frac{3}{5} \notin\langle 6\rangle_{\langle 2\rangle}$.

Recall that a ring $R$ is called presimplifiable if $Z(R) \subseteq J(R)$. A subclass of presimplifiable rings is the class of Domainlike rings where $Z(R) \subseteq N(R)$ ([4]).

Proposition 2.8. Let $R$ be a presimplifiable (Domainlike) ring. Then any r-ideal in $R$ is a J-ideal (n-ideal). 
Proof. Suppose $R$ is presimplifiable and let $I$ be an r-ideal of $R$. Let $a, b \in R$ such that $a b \in I$ where $a \notin J(R)$. Then $a \notin Z(R)$ and so clearly, Ann $(a)=0$. Since $I$ is an r-ideal, $b \in I$ and so $I$ is a J-ideal. If $R$ is Domainlike, then similarly, we conclude that any r-ideal is an n-ideal.

A proper ideal $I$ in a ring $R$ is called superfluous if whenever $J$ is an ideal of $R$ with $I+J=R$, then $J=R$.

Proposition 2.9. Any J-ideal in a ring $R$ is superfluous.

Proof. Assume $J$ is an ideal of $R$ such that $I+J=R$. Then $1=i+j$ for some $i \in I$ and $j \in J$ and so $1-j=i \in I \subseteq J(R)$. It follows that $j \in U(R)$ and hence, $J=R$ as required.

Proposition 2.10. Let $R$ be a ring and $I$ be a proper ideal of $R$. The following are equivalent.

(1) $I$ is a J-ideal of $R$.

(2) $I=(I: a)$ for every $a \notin J(R)$.

(3) For ideals $A$ and $B$ of $R, A B \subseteq I$ and $A \nsubseteq J(R)$ implies that $B \subseteq I$.

(4) $(I: a) \subseteq J(R)$ for every $a \notin I$.

Proof. (1) $\Rightarrow(2)$ Assume that $I$ is a J-ideal of $R$. For every $a \in R$, the inclusion $I \subseteq(I: a)$ always holds. Let $a \notin J(R)$ and $b \in(I: a)$. Then $a b \in I$. Since $I$ is a J-ideal, $b \in I$ and so $I=(I: a)$.

(2) $\Rightarrow$ (3) Suppose that $A B \subseteq I$ and $A \nsubseteq J(R)$ for ideals $A$ and $B$ of $R$. Since $A \nsubseteq J(R)$, there exists $a \in A$ such that $a \notin J(R)$. Then we have $a B \subseteq I$ and so $B \subseteq(I: a)=I$.

$(3) \Rightarrow(1)$ Let $a, b \in R$ with $a b \in I$ and $a \notin J(R)$. Take $A=\langle a\rangle$ and $B=\langle b\rangle$. Then $A B=\langle a\rangle\langle b\rangle=\langle a b\rangle \subseteq I$ and $A \nsubseteq J(R)$ and so $B=\langle b\rangle \subseteq I$. Hence, $b \in I$ and $I$ is a J-ideal.

$(1) \Rightarrow(4)$ Let $a \notin I$ and let $b \in(I: a)$. Then $a b \in I$. Since $I$ is a J-ideal, we get $b \in J(R)$ and the result follows.

(4) $\Rightarrow(1)$ Take $a, b \in R$ such that $a b \in I$ with $a \notin J(R)$. If $b \notin I$, then by (4), $a \in(I: b) \subseteq J(R)$ a contradiction. Thus, we have $b \in I$ and so $I$ is a $J$-ideal of $R$.

An ideal $M$ of a ring $R$ is called Jacobson if it is an intersection of maximal ideals. For example in the domain of integers any ideal generated by a product of distinct prime integers is Jacobson. Now, if $I$ is a Jacobson J-ideal, then clearly 
$J(R) \subseteq I$. Since also $I \subseteq J(R)$ by Proposition 2.2, we conclude that the only Jacobson J-ideal, if there is any, is $J(R)$.

Let $R$ be a ring and let $S$ be a nonempty subset of $R$. For an ideal $I$ of $R$, the set $(I: S)=\{r \in R: r S \subseteq I\}$ is clearly an ideal of $R$.

Lemma 2.11. Let $R$ be a ring and $S$ be a nonempty subset of $R$. If $I$ is a J-ideal of $R$ with $S \nsubseteq I$, then $(I: S)$ is a J-ideal of $R$.

Proof. If $(I: S)=R$, then $1 \in(I: S)$ and so $S \subseteq I$, a contradiction. Therefore, $(I: S)$ is a proper ideal in $R$. Let $a b \in(I: S)$ and $a \notin J(R)$. Then we have abs $\in I$ for every $s \in S$. Since $I$ is a J-ideal of $R$, we conclude that $b s \in I$ and so $b \in(I: S)$.

Definition 2.12. A J-ideal $I$ of $R$ is called a maximal $J$-ideal if there is no J-ideal which contains $I$ properly.

If an ideal $I$ of a ring $R$ is prime with $I \subseteq J(R)$, then clearly $I$ is a J-ideal. In the following proposition, we determine a condition on $I$ when the converse holds.

Proposition 2.13. Let $I$ be a maximal J-ideal of $R$. Then $I$ is a prime ideal. If, in particular, $I=J(R)$, then the converse is true.

Proof. Suppose $I$ is a maximal J-ideal. Let $a, b \in R$ such that $a b \in I$ and $a \notin I$. Then $(I: a)$ is a J-ideal by Lemma 2.11 and $I \subseteq(I: a)$. By maximality of $I$, we have $b \in(I: a)=I$ and so $I$ is a prime ideal of $R$. Now, suppose $I=J(R)$ is a prime ideal of $R$. Let $a, b \in R$ such that $a b \in I$ and $a \notin J(R)$. Then $b \in J(R)=I$ and so $I$ is a J-ideal. Moreover, $I$ is maximal by Proposition 2.2.

Next, we determine all integers $n \geq 2$ such that the ring $\mathbb{Z}_{n}$ has J-ideals.

Corollary 2.14. For any integer $n \geq 2$, the ring $\mathbb{Z}_{n}$ has J-ideals if and only if $n$ is a power of a prime integer.

Proof. $\Leftarrow)$ If $n$ is a power of a prime integer, then $\mathbb{Z}_{n}$ is quasi-local and the result follows by Proposition 2.3.

$\Rightarrow$ Suppose $\mathbb{Z}_{n}$ has a J-ideal. Suppose that $n=p_{1}^{t_{1}} p_{2}^{t_{2}} \ldots p_{k}^{t_{k}}$ is a distinct prime decomposition of $n$ where $k \geq 2$. Then $J\left(\mathbb{Z}_{n}\right)=\left\langle p_{1} p_{2} \ldots p_{k}\right\rangle$ is not a J-ideal of $\mathbb{Z}_{n}$ since it is not prime. Let $I$ be a maximal J-ideal of $\mathbb{Z}_{n}$. Then $I \subseteq J\left(\mathbb{Z}_{n}\right)$ and by Proposition 2.13, $I$ is also a prime ideal. Therefore, $I=J\left(\mathbb{Z}_{n}\right)$ which is a contradiction. 
We recall that a ring $R$ is called semiprimitive if $J(R)=0$. Moreover, $R$ is called a von Neumann regular ring if for every $a \in R$, there exists $x \in R$ such that $a=a^{2} x$. It is well known that the ring of integers and a von Neumann regular ring are semiprimitive. The proof of the following proposition can be achieved simply by using Proposition 2.2 .

Proposition 2.15. Let $R$ be a ring.

(1) If $R$ is a semiprimitive ring which is not an integral domain, then $R$ has no J-ideals.

(2) Let $R$ be a semiprimitive ring. Then $R$ is an integral domain if and only if the zero ideal is a J-ideal of $R$.

Proposition 2.16. Let $R$ be a ring. Then the following statements are equivalent.

(1) $R$ is a field.

(2) $R$ is a von Neumann regular ring and 0 is an $n$-ideal in $R$.

(3) $R$ is a von Neumann regular ring and 0 is an $J$-ideal in $R$.

Proof. $(1) \Rightarrow(2)$ Theorem 2.15 in [13].

$(2) \Rightarrow(3)$ Clear by Proposition 2.4.

$(3) \Rightarrow(1)$ Suppose that $R$ is a von Neumann regular ring and 0 is a J-ideal. Let $0 \neq a \in R$. Since $R$ is von Neumann regular, $a=a^{2} x$ for some $x \in R$. Now, $a(1-a x)=0$ and $a \neq 0=J(R)$ imply that $a x=1$ and thus $a$ is unit. Consequently, $R$ is a field.

It is well known that if $R$ is an integral domain, then $J(R[x])=0$ and so, $R[x]$ is a semiprimitive integral domain. By Proposition 2.15, we conclude that the zero ideal of $R[x]$ is the only J-ideal.

Lemma 2.17. [11] Let $R$ be ring. Then $J(R[|x|])=J(R)+x R[|x|]$.

Proposition 2.18. Let $R$ be a ring and $I$ be a proper ideal of $R$. Then $I[|x|]$ is a $J$-ideal of $R[|x|]$ if and only if $I$ is a J-ideal of $R$.

Proof. $\Leftarrow)$ Suppose $I$ is a J-ideal of $R$. Let $f(x)=\sum_{i=0}^{\infty} a_{i} x^{i}, g(x)=\sum_{i=0}^{\infty} b_{i} x^{i} \in$ $R[[x]]$ such that $f(x) g(x) \in I[|x|]$ and $f(x) \notin J(R[|x|])$. Then $\sum_{i=0}^{\infty}\left(\sum_{p+q=i} a_{p} b_{q}\right) x^{i} \in$ $I[|x|]$ and so $\sum_{p+q=i} a_{p} b_{q} \in I$. Now, $a_{0} b_{0} \in I$ with $a_{0} \notin J(R)$ by Lemma 2.17. Thus, $b_{0} \in I$ as $I$ is a J-ideal in $R$. Also, $a_{0} b_{1}+a_{1} b_{0} \in I$ implies that $a_{0} b_{1} \in I$ and so again $b_{1} \in I$. Continuing this process, we get $g(x)=\sum_{i=0}^{\infty} b_{i} x^{i} \in I[|x|]$ and so $I[|x|]$ is a J-ideal of $R[|x|]$. 
$\Rightarrow)$ Suppose $I[|x|]$ is a J-ideal in $R[|x|]$ and let $a, b \in R$ such that $a b \in I$ with $a \notin J(R)$. Then again by Lemma 2.17, $a b \in I[|x|]$ with $a \notin J(R[|x|])$. Thus, $b \in I[|x|]$ and so $b \in I$ as required.

Recall that for an ideal $I$ of a ring $R$, the Jacobson radical of $I$ (denoted by $J(I)$ ) is defined as the intersection of all maximal ideals of $R$ containing $I$. The following properties can be easily verified for any ideals $I$ and $K$ of $R$ :

(1) $I \subseteq \sqrt{I} \subseteq J(I)$.

(2) $I \subseteq K$ implies that $J(I) \subseteq J(K)$.

(3) $J(R) \subseteq J(I)$.

(4) $J(J(I))=J(I)$.

(5) $J(I) / I=J(R / I)$.

Definition 2.19. Let $I$ be a proper ideal of a ring $R$. Then, $I$ is called $J$-primary if whenever $a, b \in R, a b \in I$ implies that $a \in J(I)$ or $b \in I$.

In the following proposition, we prove that J-ideals and J-primary ideals that contained in the Jacobson radical are the same.

Proposition 2.20. Let $I$ be an ideal of a ring $R$ such that $I \subseteq J(R)$. Then $I$ is a $J$-ideal if and only if $I$ is J-primary.

Proof. Let $R$ be a ring, $I$ be a proper ideal of $R$ and $a, b \in R$.

$\Rightarrow)$ Suppose $I$ is a J-ideal in $R$ and let $a b \in I$ with $a \notin J(I)$. Since $J(R) \subseteq J(I)$, we conclude that $a \notin J(R)$. Now, $I$ is a J-ideal implies that $b \in I$ and so, $I$ is a J-primary ideal of $R$.

$\Leftarrow)$ Let $I$ be a J-primary ideal of $R$ and let $a b \in I$ with $a \notin J(R)$. Now, $I \subseteq J(R)$ implies that $J(I) \subseteq J(J(R))=J(R)$. Thus, $a \notin J(I)$ and so $b \in I$ as $I$ is J-primary. Therefore, $I$ is a J-ideal.

Proposition 2.21. Let $R$ be a ring and $K$ be an ideal of $R$ with $K \nsubseteq J(R)$. Then the following hold.

(1) If $I_{1}$ and $I_{2}$ are $J$-ideals of $R$ with $I_{1} K=I_{2} K$, then $I_{1}=I_{2}$.

(2) If $I$ is an ideal such that $I K$ is a J-ideal, then $I K=I$.

Proof. (1) We have $I_{2} K=I_{1} K \subseteq I_{1}$. Since $I_{1}$ is a J-ideal, $I_{2} \subseteq I_{1}$ by Proposition 2.10. Similarly, as $I_{2}$ is a J-ideal, we get $I_{1} \subseteq I_{2}$ and the equality holds.

(2) Since $I K$ is a J-ideal, $I K \subseteq I K$ and $K \nsubseteq J(R)$, we get $I \subseteq I K$. The other inclusion is trivial.

The proof of the following lemma is straightforward. 
Lemma 2.22. Let $R$ and $S$ be two rings. If $f$ is a ring epimorphism from $R$ onto $S$, then $f(J(R)) \subseteq J(S)$.

Proposition 2.23. If $f: R \rightarrow S$ is a ring epimorphism, then the following hold.

(1) If $I$ is a J-ideal of $R$ with $\operatorname{ker}(f) \subseteq I$, then $f(I)$ is a J-ideal of $S$.

(2) If $K$ is a J-ideal of $S$ with $\operatorname{ker}(f) \subseteq J(R)$, then $f^{-1}(K)$ is a J-ideal of $R$.

Proof. (1) Let $c, d \in S$ such that $c d \in f(I)$ and $c \notin J(S)$. As $f$ is an epimorphism, we can choose $a, b \in R$ such that $c=f(a)$ and $d=f(b)$. Then $c d=f(a b) \in f(I)$. Since ker $f \subseteq I$, we conclude that $a b \in I$. Also, note that $a \notin J(R)$ since otherwise if $a \in J(R)$, then $f(a)=c \in J(S)$ by Lemma 2.22 which is a contradiction. Since $I$ is a J-ideal of $R$, we conclude that $b \in I$ and so $d=f(b) \in f(I)$ as required.

(2) Let $a, b \in R$ where $a b \in f^{-1}(K)$ and $a \notin J(R)$. Then $f(a) f(b)=f(a b) \in K$. We prove that $f(a) \notin J(S)$. Suppose $f(a) \in J(S)$ and let $M$ be a maximal ideal of $R$. Then $f(M)$ is a maximal ideal of $S$ since $\operatorname{ker}(f) \subseteq J(R) \subseteq M$. Thus, $f(a) \in f(M)$ and so $a \in M$ as $\operatorname{ker}(f) \subseteq M$. Hence, $a \in J(R)$ which is a contradiction. Since $K$ is a J-ideal, $f(b) \in K$ and so $b \in f^{-1}(K)$. It follows that $f^{-1}(K)$ is a J-ideal of $R$.

Corollary 2.24. Let $R$ be a ring and let $I, K$ be two ideals of $R$ with $K \subseteq I$. Then the followings hold.

(1) If $I$ is a J-ideal of $R$, then $I / K$ is a J-ideal of $R / K$.

(2) If $I / K$ is a J-ideal of $R / K$ and $K \subseteq J(R)$, then $I$ is a J-ideal of $R$.

(3) If $I / K$ is a J-ideal of $R / K$ and $K$ is a J-ideal of $R$, then $I$ is a J-ideal of $R$.

Proof. (1) Assume that $I$ is a J-ideal of $R$ with $K \subseteq I$. Let $\pi: R \rightarrow R / K$ be the natural epimorphism defined by $\pi(R)=r+K$. Note that $\operatorname{ker} \pi=K \subseteq I$. Thus, by Proposition 2.23 (1), it follows that $\pi(I)=I / K$ is a J-ideal of $R / K$.

(2) Again consider the natural epimorphism $\pi: R \rightarrow R / K$. Since $K \subseteq J(R)$, by Proposition $2.23(2), I=\pi^{-1}(I / K)$ is a J-ideal of $R$.

(3) This is clear by (2) and Proposition 2.2 .

The converse of (1) of Corollary 2.24 is not true in general. For example, $\langle 0\rangle \bumpeq$ $\langle 5\rangle /\langle 5\rangle$ is a J-ideal in the field $\mathbb{Z} /\langle 5\rangle \simeq \mathbb{Z}_{5}$ but $\langle 5\rangle$ is not a J-ideal of $\mathbb{Z}$ as $\langle 5\rangle \nsubseteq$ $J(\mathbb{Z})=\langle 0\rangle$.

Proposition 2.25. Let $R$ be a ring and $\left\{I_{i}: i \in \Delta\right\}$ be a nonempty family of $J$ ideals of $R$. Then $\bigcap_{i \in \Delta} I_{i}$ is a J-ideal of $R$. 
Proof. Let $a, b \in R$ such that $a b \in \bigcap_{i \in \Delta} I_{i}$ and $a \notin J(R)$. Then $a b \in I_{i}$ for all $i \in \Delta$. Since $I_{i}$ is a J-ideal for all $i \in \Delta$, we conclude that $b \in I_{i}$ for all $i$ and so $b \in \bigcap_{i \in \Delta} I_{i}$. Therefore, $\bigcap_{i \in \Delta} I_{i}$ is a J-ideal.

Proposition 2.26. Let $R$ be a ring and $S$ be a multiplicatively closed subset of $R$ such that $J\left(S^{-1} R\right)=S^{-1} J(R)$. If $I$ is a J-ideal of $R$ with $I \cap S=\phi$, then $S^{-1} I$ is a J-ideal of $S^{-1} R$.

Proof. Let $a, b \in R$ and $s, t \in S$ such that $\frac{a}{s} \cdot \frac{b}{t} \in S^{-1} I$ and $\frac{a}{s} \notin J\left(S^{-1} R\right)$. Then $\frac{a}{s} \notin S^{-1} J(R)$ and so clearly $a \notin J(R)$. Now, $\frac{a b}{s t}=\frac{x}{v}$ for some $x \in I$ and $v \in S$ and so there exists $u \in S$ such that $u a b \in I$. Since $I$ is a J-ideal of $R, u b \in I$ and so $\frac{b}{t}=\frac{u b}{u t} \in S^{-1} I$. It follows that $S^{-1} I$ is a J-ideal of $S^{-1} R$.

We note that the converse of Proposition 2.26 need be true. For example while $\langle 2\rangle_{\langle 2\rangle}$ is a J-ideal in the ring $\mathbb{Z}_{\langle 2\rangle}$, the ideal $\langle 2\rangle$ is not a J-ideal of $\mathbb{Z}$ as $\langle 2\rangle \nsubseteq J(\mathbb{Z})$.

Definition 2.27. Let $S$ be a nonempty subset of a ring $R$ with $R-J(R) \subseteq S$. Then $S$ is called a J-multiplicatively closed subset of $R$ if $a b \in S$ for all $a \in R-J(R)$ and all $b \in S$.

In the following proposition, we determine the relation between J-ideals and J-multiplicatively closed subsets of $R$.

Proposition 2.28. For a proper ideal $I$ of $R, I$ is a $J$-ideal of $R$ if and only if $R-I$ is a J-multiplicatively closed subset of $R$.

Proof. Suppose that $I$ is a J-ideal of $R$. Then by Proposition 2.2, we have $I \subseteq J(R)$ and so $R-J(R) \subseteq R-I$. Let $a \in R-J(R)$ and $b \in R-I$. Assume that $a b \in I$. Since $a \notin J(R)$ and $I$ is a J-ideal, we get $b \in I$, a contradiction. Thus, we conclude that $a b \in R-I$ and so $R-I$ is a $J$-multiplicatively closed subset of $R$. For the converse, suppose that $I$ is an ideal and $R-I$ is a J-multiplicatively closed subset of $R$. We prove that $I$ is a J-ideal. Let $c, d \in R$ and $c d \in I$ with $c \notin J(R)$. Then we have $d \in I$ since otherwise we would have $c d \in R-I$ which is contradiction. So, $I$ is a J-ideal of $R$.

Recall that if $I$ is an ideal which is disjoint from a multiplicatively closed subset $S$ of $R$, then there exists a prime ideal $P$ of $R$ containing $I$ such that $P \cap S=\emptyset$. The following proposition states a similar result for J-ideals.

Proposition 2.29. Let $I$ be an ideal of $R$ such that $I \cap S=\emptyset$ where $S$ is a Jmultiplicatively closed subset of $R$. Then there exists a J-ideal $K$ containing I such that $K \cap S=\emptyset$. 
Proof. Consider the set $\Omega=\{Q: Q$ is an ideal of $R$ with $Q \cap S=\emptyset\}$. Then $\Omega$ is partially ordered by inclusion. Since $I \in \Omega$, we have $\Omega \neq \emptyset$. If $Q_{1} \subseteq Q_{2} \subseteq \cdots$ is any

chain in $\Omega$, then $\bigcup_{i=1}^{\infty} Q_{i}$ is an ideal of $R$ with $\left(\bigcup_{i=1}^{\infty} Q_{i}\right) \cap S=\emptyset$. Hence, $\bigcup_{i=1}^{\infty} Q_{i}$ is an upper bound of the chain. By using Zorn's Lemma, we get a maximal element $K$ of $\Omega$. Now, we show that $K$ is a J-ideal of $R$. Suppose not. Then we have $x y \in K$ for some $x \notin J(R)$ and $y \notin K$. Thus, we get $y \in(K: x)$ and $K \varsubsetneqq(K: x)$. By the maximality of $K$, we have $(K: x) \cap S \neq \emptyset$ and so there exists an $s \in S$ such that $s \in(K: x)$. So, we have $x s \in K$. Also, $s x \in S$ because $x \in R-J(R), s \in S$ and $S$ is a J-multiplicatively closed subset of $R$. Thus, we have $S \cap K \neq \emptyset$ and this contradicts that $K \in \Omega$. Hence, $K$ is a J-ideal of $R$.

Recall that if $R_{1}$ and $R_{2}$ are rings, then $\left(0,1_{R_{2}}\right),\left(1_{R_{1}}, 0\right) \notin J\left(R_{1} \times R_{2}\right)$. Indeed, if, say $(1,0) \in J\left(R_{1} \times R_{2}\right)$, then $(0,1)=(1,1)-(1,0) \in U\left(R_{1} \times R_{2}\right)$ which is impossible.

Proposition 2.30. Let $R_{1}$ and $R_{2}$ be two rings. Then $R_{1} \times R_{2}$ has no J-ideals.

Proof. Assume that $I=I_{1} \times I_{2}$ is a J-ideal of $R_{1} \times R_{2}$ where $I_{1}, I_{2}$ are ideals of $R_{1}, R_{2}$ respectively. Since $(0,1)(1,0) \in I_{1} \times I_{2}$ with $(0,1),(1,0) \notin J\left(R_{1} \times R_{2}\right)$, we get $(0,1),(1,0) \in I_{1} \times I_{2}=I$ and so $I=R_{1} \times R_{2}$, a contradiction.

\section{J-submodules over commutative rings}

In this section, we generalize the concept of J-ideal to submodules of $R$-modules. We first clarify some analogous results to those of J-ideals with a focus on multiplication modules. Next, we study J-ideals in the idealization ring of modules in a ring $R$ and investigate some examples and properties concerning them.

For an $R$-module $M$ and a submodule $N$ of $M$, the residual ideal in $R$ of $N$ by $M$ is defined as $(N: M)=\{r \in R: r m \in N$ for all $m \in M\}$. Moreover, for an ideal $I$ of $R$ and a submodule $N$ of $M$, the set $\left(N:_{M} I\right)=\{m \in M: a m \in N$ for all $a \in I\}$ is a submodule of $M$.

Definition 3.1. Let $M$ be an $R$-module. Then a proper submodule $N$ of $M$ is called $a J$-submodule if for all $a \in R$ and $m \in M$, whenever $a m \in N$ with $a \notin(J(R) M: M)$, then $m \in N$.

Clearly, if $(J(R) M: M)=R$, then our definition implies that any proper submodule of $M$ is a J-submodule. However, by Nakayama's Lemma, the only finitely generated $R$-module $M$ such that $(J(R) M: M)=R$ is the trivial module $\{0\}$. 
Proposition 3.2. Let $M$ be an $R$-module, $N$ be a submodule of $M$ and $I$ be an ideal of $R$.

(1) If $N$ is a J-submodule of $M$ and $(J(R) M: M)=J(R)$, then $(N: M)$ is a $J$-ideal of $R$.

(2) If $N$ is a J-submodule of $M$, then $\left(N:_{M} I\right)$ is also a J-submodule of $M$.

Proof. (1) Let $a b \in(N: M)$ where $a, b \in R$ and $a \notin J(R)$. Then we have $a b M \subseteq N$ and so $a b m \in N$ for all $m \in M$. Since $N$ is a J-submodule of $M$ and $a \notin(J(R) M: M), b m \in N$ for all $m \in M$. Thus, $b M \subseteq N$ and so $b \in(N: M)$. Therefore, $(N: M)$ is a J-ideal of $R$.

(2) Let $r m \in\left(N:_{M} I\right)$ where $r \in R$ and $m \in M$ with $r \notin(J(R) M: M)$. Then we have $r m I \subseteq N$ and so $r m i \in N$ for all $i \in I$. Since $N$ is a J-submodule of $M$ and $r \notin(J(R) M: M), m i \in N$ for all $i \in I$. Thus, $m I \subseteq N$ and so $m \in\left(N:_{M} I\right)$. Therefore, $\left(N:_{M} I\right)$ is a $\mathrm{J}$-submodule of $M$.

If $(J(R) M: M) \nsubseteq J(R)$, then (1) of Proposition 3.2 need not be true. For example, consider the $\mathbb{Z}$-module $M=\mathbb{Z}_{2}$. Then $2 \mathbb{Z}=(J(\mathbb{Z}) M: M) \nsubseteq J(\mathbb{Z})=\{0\}$. Now, $N=\{\overline{0}\}$ is clearly, a J-submodule of $\mathbb{Z}_{2}$ but $2 \mathbb{Z}=(N: M)$ is not a J-ideal of $\mathbb{Z}$.

We recall that an $R$-module $M$ is called multiplication, [8] if each submodule of $M$ is of the form $I M$ for some ideal $I$ of $R$. Equivalently, $M$ is multiplication if and only if $N=(N: M) M$ for every submodule $N$ of $M$. Multiplication modules has an important place in theory of modules and it has been widely studied in many papers. See, for example [3] and [12]. Moreover, $M$ is called faithful if $\left(0_{M}: M\right)=\{0\}$, the zero ideal of $R$. It is well known that if $M$ is a finitely generated faithful multiplication $R$-module, then $(I M: M)=I$ for any ideal $I$ of $R([8])$.

Proposition 3.3. Let $N$ be a submodule of a multiplication module $M$ such that $(N: M)$ is a J-ideal of $R$. Then $N$ is a J-submodule of $M$.

Proof. Let $r \in R$ and $m \in M$ such that $r m \in N$ and $r \notin(J(R) M: M)$. Then $r(\langle m\rangle: M) \subseteq(\langle r m\rangle: M) \subseteq(N: M)$. Since $J(R) \subseteq(J(R) M: M), r \notin J(R)$ and so $(\langle m\rangle: M) \subseteq(N: M)$ as $(N: M)$ is a J-ideal of $R$. Now, $M$ is a multiplication module implies that $\langle m\rangle=(\langle m\rangle: M) M \subseteq(N: M) M=N$. Thus, $m \in N$ and $N$ is a J-submodule of $M$.

Corollary 3.4. Let $M$ be a finitely generated faithful multiplication R-module and $N$ be a proper submodule of $M$. The following are equivalent. 
(1) $N$ is a J-submodule of $M$.

(2) $(N: M)$ is a J-ideal of $R$.

(3) $N=L M$ where $L$ is a J-ideal of $R$.

Proof. (1) $\Leftrightarrow(2)$ Follows by Propositions 3.2 and 3.3 and the fact that (IM : $M)=I$ for any ideal $I$ of $R$.

$(2) \Leftrightarrow(3)$ We just choose $L=(N: M)$.

In the following proposition, we give a characterization of J-submodules.

Proposition 3.5. Let $M$ be an R-module and $N$ be a proper submodule of $M$. Then $N$ is a J-submodule of $M$ if and only if for any ideal $I$ of $R$ and every submodule $K$ of $M$, we have $I K \subseteq N$ with $I \nsubseteq(J(R) M: M)$ implies $K \subseteq N$.

Proof. $\Rightarrow)$ Suppose $I K \subseteq N$ and $I \nsubseteq(J(R) M: M)$. Let $a \in I$ with $a \notin$ $(J(R) M: M)$ and $k \in K$. Since $a k \in N$ and $N$ is a J-submodule of $M, k \in N$. It follows that $K \subseteq N$ as needed.

$\Leftarrow)$ Let $r \in R$ and $m \in M$ such that $r m \in N$ and $r \notin(J(R) M: M)$. Then clearly $\langle r\rangle\langle m\rangle \subseteq N$ with $\langle r\rangle \nsubseteq(J(R) M: M)$. By assumption, we conclude that $\langle m\rangle \subseteq N$ and so $m \in N$.

If $M$ is a multiplication $R$-module and $N=I M, K=J M$ are two submodules of $M$, then the product $N K$ of $N$ and $K$ is defined as $N K=(I M)(J M)=(I J) M$. In particular, if $m_{1}, m_{2} \in M$, then $m_{1} m_{2}=\left\langle m_{1}\right\rangle\left\langle m_{2}\right\rangle$.

Recall that the Jacobson radical $J(M)$ of an $R$-module $M$ is the intersection of all maximal submodules of $M$. Similarly, the prime radical $\operatorname{rad}\left(0_{M}\right)$ of $M$ is the intersection of all prime submodules of $M$. Since every maximal submodule of $M$ is prime, clearly, $\operatorname{rad}\left(0_{M}\right) \subseteq J(M)$. If $M$ is finitely generated faithful multiplication, then $J(M)=J(R) M([8])$.

Proposition 3.6. Let $M$ be a finitely generated faithful multiplication R-module. If $N$ is a $J$-submodule of $M$, then $N \subseteq J(M)$.

Proof. We note that $J(M)=J(R) M$. Suppose $N \nsubseteq J(M)$. Then clearly, $(N$ : $M) \nsubseteq(J(R) M: M)=J(R)$. But $(N: M)$ is a J-ideal by Proposition 3.2 which contradicts Proposition 2.2. Thus, $N \subseteq J(M)$ as required.

In [13], the concept of n-submodules has been defined. A submodule $N$ of an $R$ module $M$ is called an n-submodule of $M$ if whenever $a \in R, m \in M$ and $a m \in N$ with $a \notin N i l(M)$, then $m \in N$ where $\operatorname{Nil}(M)=\sqrt{(0: M)}$. 
Proposition 3.7. Let $M$ be an $R$-module satisfying $J(M) \subseteq J(R) M$. Then any $n$-submodule of $M$ is a $J$-submodule.

Proof. We first prove that $N i l(M)=\sqrt{(0: M)} \subseteq(\operatorname{Rad}(0): M) \subseteq(J(M): M) \subseteq$ $(J(R) M: M)$. Let $r \in \sqrt{(0: M)}$. Let $Q$ be a prime submodule of $M$. Then $(Q: M)$ is a prime ideal of $R$ and clearly $(0: M) \subseteq(Q: M)$. Thus, $r \in(Q: M)$ and then $r M \subseteq Q$ for all prime submodules $Q$ of $M$. It follows that $r M \subseteq \operatorname{rad}(0)$ and so $r \in(\operatorname{Rad}(0): M)$. Since any maximal submodule of $M$ is prime, $(\operatorname{rad}(0): M) \subseteq$ $(J(M): M) \subseteq(J(R) M: M)$. Now, let $a \in R$ and $m \in M$ such that $a m \in N$ where $N$ is an n-submodule of $M$. If $a \notin(J(R) M: M)$, then $a \notin \sqrt{(0: M)}=\operatorname{Nil}(M)$. Thus, $m \in N$ and $N$ is a J-submodule of $M$.

Corollary 3.8. Any n-submodule of a finitely generated faithful multiplication module is a J-submodule.

Proposition 3.9. Let $N$ be a proper submodule of a finitely generated faithful multiplication R-module $M$. Then $N$ is a J-submodule of $M$ if and only if whenever $K$ and $L$ are submodules of $M$ such that $K L \subseteq N$ and $K \nsubseteq J(M)$, then $L \subseteq N$.

Proof. $\Rightarrow)$ Suppose $K=I M$ and $L=J M$ where $I$ and $J$ are ideals of $R$. Suppose on contrary that $L \nsubseteq N$ and choose $x=\sum_{i=1}^{n} r_{i} m_{i} \in K-J(R) M$ and $y=\sum_{i=1}^{k} a_{i} t_{i} \in$ $L-N$ where $r_{i} \in I, a_{i} \in J$ and $m_{i}, t_{i} \in M$ for all $i$. Then $r_{j} \notin J(R)$ for some $j \in\{1,2, \ldots, n\}$. Now, $r_{j} y \in I J M=K L \subseteq N$ with $r_{j} \notin(J(R) M: M)$. Since $N$ is a J-submodule, $y \in N$, a contradiction. Therefore, $L \subseteq N$ as required.

$\Leftarrow)$ By Corollary 3.4, it is enough to prove that $(N: M)$ is a J-ideal of $R$. Let $a b \in(N: M)$ where $a, b \in R$ and $a \notin J(R)$. Let $K=\langle a\rangle M$ and $L=\langle b\rangle M$. Then $K L=\langle a\rangle\langle b\rangle M \subseteq N$. Moreover, $K \nsubseteq J(M)=J(R) M$ since otherwise $\langle a\rangle=$ $(\langle a\rangle M: M)=(K: M) \subseteq(J(R) M: M)=J(R)$, a contradiction. By assumption, we have $L \subseteq N$ and so $\langle b\rangle M \subseteq N$. This gives $b \in(N: M)$ and $(N: M)$ is a J-ideal of $R$.

Corollary 3.10. Let $N$ be a proper submodule of a finitely generated faithful multiplication R-module $M$. Then $N$ is a J-submodule of $M$ if and only if whenever $m_{1}, m_{2} \in M$ such that $m_{1} m_{2} \in N$ and $m_{1} \notin J(M)$, then $m_{2} \in N$.

Proposition 3.11. Let $f: M \longrightarrow N$ be an R-module epimorphism. Then

(1) If $P$ is a J-submodule of $M$ with $\operatorname{ker}(f) \subseteq P$, then $f(P)$ is a J-submodule of $N$.

(2) If $Q$ is a J-submodule of $N$ with $\operatorname{ker}(f) \subseteq J(R) M$, then $f^{-1}(Q)$ is a $J$-submodule of $M$. 
Proof. (1) Let $r \in R$ and $n \in N$ such that $r n \in f(P)$ with $r \notin(J(R) N: N)$. Choose $m \in M$ such that $f(m)=n$. Then $r n=r f(m)=f(R m) \in f(P)$. Thus, $f(R m-a)=0$ for some $a \in P$ and so $r m-a \in \operatorname{ker}(f) \subseteq P$. It follows that $r m \in P$. Moreover, we have $r \notin(J(R) M: M)$. Indeed, if $r M \subseteq J(R) M$, then $r N=r f(M)=f(r M) \subseteq f(J(R) M)=J(R) f(M)=J(R) N$ which is a contradiction. Since $P$ is a J-submodule, $m \in P$ and so $n=f(m) \in f(P)$ as required.

(2) Let $r \in R$ and $m \in M$ such that $r m \in f^{-1}(Q)$ and $r \notin(J(R) M: M)$. Then $r f(m)=f(R m) \in Q$. We prove that $r \notin(J(R) N: N)$. Suppose on contrary that $r N \subseteq J(R) N$. Then $r f(M) \subseteq J(R) f(M)$ and so $f(R M) \subseteq f(J(R) M)$. Now, if $x \in r M$, then $f(x) \in f(R M) \subseteq f(J(R) M)$ and hence $x-t \in \operatorname{ker}(f) \subseteq J(R) M$ for some $t \in J(R) M$. It follows that $x \in J(R) M$ and $r M \subseteq J(R) M$ which is a contradiction. Since $Q$ is a J-submodule of $N, f(m) \in Q$ and so $m \in f^{-1}(Q)$.

Let $R$ be a ring and $M$ be an $R$-module. The idealization $\operatorname{ring} R(M)$ of $M$ in $R$ is defined as the set $\{(r, m): r \in R, m \in M\}$ with the usual componentwise addition and multiplication defined as $(r, m)(s, n)=(r s, r n+s m)$. It can be easily verified that $R(M)$ is a commutative ring with identity $\left(1_{R}, 0_{M}\right)$. If $I$ is an ideal of $R$ and $N$ is a submodule of $M$, then $I(N)=\{(r, m): r \in I, m \in N\}$ is an ideal of $R(M)$ if and only if $I M \subseteq N$. In this case, $I(N)$ is called a homogeneous ideal of $R(M)$, see [2]. In [2], the Jacobson radical of $R(M)$ is characterized as $J(R(M))=J(R)(M)$.

Proposition 3.12. Let $I$ be a J-ideal of $R, M$ be an $R$-module and $N$ be a submodule of $M$. Then

(1) $I(M)$ is a J-ideal of $R(M)$.

(2) If $(J(R) M: M)=J(R)$ and $N$ is a J-submodule of $M$ with $I M \subseteq N$, then $I(N)$ is a $J$-ideal of $R(M)$.

Proof. (1) Let $\left(R_{1}, m_{1}\right),\left(R_{2}, m_{2}\right) \in R(M)$ such that $\left(R_{1}, m_{1}\right)\left(R_{2}, m_{2}\right) \in I(M)$ with $\left(R_{1}, m_{1}\right) \notin J(R(M))$. Then we have $r_{1} r_{2} \in I$ and $r_{1} \notin J(R)$. Since $I$ is a J-ideal of $R$, we conclude that $r_{2} \in I$ and so $\left(R_{2}, m_{2}\right) \in I(M)$. Consequently, $I(M)$ is a J-ideal of $R(M)$.

(2) Let $\left(R_{1}, m_{1}\right),\left(R_{2}, m_{2}\right) \in R(M)$ such that $\left(R_{1}, m_{1}\right)\left(R_{2}, m_{2}\right) \in I(N)$ and $\left(R_{1}, m_{1}\right) \notin J(R(M))=J(R)(M)$. Then we have $r_{1} r_{2} \in I$ and $r_{1} \notin J(R)$. As $I$ is a J-ideal of $R$, then we have $r_{2} \in I$ and so $r_{2} m_{1} \in I M \subseteq N$. Since $r_{1} m_{2}+r_{2} m_{1} \in N$, $r_{1} m_{2} \in N$. But, $r_{1} \notin(J(R) M: M)$ and so $m_{2} \in N$ as $N$ is a J-submodule of $M$. Therefore, $\left(R_{2}, m_{2}\right) \in I(N)$ and $I(N)$ is a J-ideal of $R(M)$. 
If $I$ is a J-ideal of a ring $R$ and $N$ is a submodule of an $R$-module $M$ with $I M \subseteq N$, then $I(N)$ need not be a J-ideal of $R(M)$. For example, $\{0\}$ is a Jideal of the ring of integers $\mathbb{Z}$ and $\overline{0}$ is a submodule of the $\mathbb{Z}$-module $\mathbb{Z}_{6}$. But $0(\overline{0})$ is not a J-ideal of $\mathbb{Z}\left(\mathbb{Z}_{6}\right)$ since $(2, \overline{0})(0, \overline{3}) \in 0(\overline{0})$ and $(2, \overline{0}) \notin J\left(\mathbb{Z}\left(\mathbb{Z}_{6}\right)\right)$ but $(0, \overline{3}) \notin 0(\overline{0})$. Also, the condition $(J(R) M: M)=J(R)$ in Proposition $3.12(2)$ can not be discarded. For example, consider the $\mathbb{Z}$-module $\mathbb{Z}_{2}$. Put $I=(0)$ and $N=(\overline{0})$. Then $I$ is a J-ideal of $\mathbb{Z}$ and $N$ is a J-submodule of $\mathbb{Z}_{2}$. Also note that $(J(R) M: M)=(2) \neq J(R)=(0)$. However, $I(N)$ is not a J-ideal of $\mathbb{Z}\left(\mathbb{Z}_{2}\right)$ because $(2, \overline{0})(0, \overline{1}) \in I(N),(2, \overline{0}) \notin J(R) M$ and $(0, \overline{1}) \notin I(N)$.

Corollary 3.13. Let $M$ be a finitely generated faithful multiplication R-module. If $I$ is a J-ideal of $R$ and $N$ is a J-submodule of $M$ with $I M \subseteq N$, then $I(N)$ is a $J$-submodule of $R(M)$.

Proposition 3.14. Let $I$ be an ideal of a ring $R$ and $N$ a proper submodule of an $R$-module $M$. If $I(N)$ is a J-ideal of $R(M)$, then $I$ is a J-ideal of $R$ and $N$ is a $J$-submodule of $M$.

Proof. Suppose that $I(N)$ is a J-ideal of $R(M)$. First, we show that $I$ is a J-ideal of $R$. Let $a b \in I$ with $a \notin J(R)$. Then we have $\left(a, 0_{M}\right)\left(b, 0_{M}\right)=\left(a b, 0_{M}\right) \in I(N)$ with $\left(a, 0_{M}\right) \notin J(R)(M)=J(R(M))$. Since $I(N)$ is a J-ideal of $R(M)$, we conclude that $\left(b, 0_{M}\right) \in I(N)$ and so $b \in I$. Now, we show that $N$ is a J-submodule of $M$. Let $a m \in N$ with $a \notin(J(R) M: M)$. Then we have $\left(a, 0_{M}\right)(0, m)=(0, a m) \in I(N)$ with $\left(a, 0_{M}\right) \notin J(R(M))$. Since $I(N)$ is a J-ideal of $R(M)$, we conclude that $(0, m) \in I(N)$ and so $m \in N$, as needed.

Acknowledgement. The authors would like to thank the referee for careful reading our manuscript and his/her valuable comments which improved this work.

\section{References}

[1] D. D. Anderson and M. Bataineh, Generalizations of prime ideals, Comm. Algebra, 36(2) (2008), 686-696.

[2] D. D. Anderson and M. Winders, Idealization of a module, J. Commut. Algebra, 1(1) (2009), 3-56.

[3] D. D. Anderson, T. Arabaci, U. Tekir and S. Koc, On S-multiplication modules, Comm. Algebra, 48(8) (2020), 3398-3407.

[4] D. D. Anderson, M. Axtell, S. J. Forman and J. Stickles, When are associates unit multiples, Rocky Mountain J. Math., 34(3) (2004), 811-828. 
[5] A. Badawi, U. Tekir and E. Yetkin, On 2-absorbing primary ideals in commutative rings, Bull. Korean Math. Soc., 51(4) (2014), 1163-1173.

[6] S. Ebrahimi Atani and F. Farzalipour, On weakly primary ideals, Georgian Math. J., 12(3) (2005), 423-429.

[7] M. Ebrahimpour and R. Nekooei, On generalizations of prime ideals, Comm. Algebra, 40(4) (2012), 1268-1279.

[8] Z. A. El-Bast and P. F. Smith, Multiplication modules, Comm. Algebra, 16(4) (1988), 755-779.

[9] H. A. Khashan, On almost prime submodules, Acta Math. Sci. Ser. B (Engl. Ed.), 32(2) (2012), 645-651.

[10] R. Mohamadian, r-Ideals in commutative rings, Turkish J. Math., 39 (2015), 733-749.

[11] R. Y. Sharp, Steps in Commutative Algebra, Second edition, London Mathematical Society Student Texts, 51, Cambridge University Press, Cambridge, 2000.

[12] P. F. Smith, Some remarks on multiplication modules, Arch. Math. (Basel), 50(3) (1988), 223-235.

[13] U. Tekir, S. Koc and K. H. Oral, n-Ideals of commutative rings, Filomat, 31(10) (2017), 2933-2941.

Hani A. Khashan (Corresponding Author) and Amal B. Bani-Ata

Department of Mathematics

Faculty of Science

Al al-Bayt University

Al Mafraq, Jordan

e-mails: hakhashan@aabu.edu.jo (H. A. Khashan)

amalnov@yahoo.com (A. B. Bani-Ata) 